\title{
A Comparison of xylometazoline (Otrivine) and phenylephrine/lignocaine mixture (Cophenylcaine) for the purposes of rigid nasendoscopy: a prospective, double-blind, randomised trial
}

\author{
N A McCluney, C Y Eng, M S W Lee, L G McClymont
}

\begin{abstract}
Objective: To evaluate if phenylephrine-lignocaine mixture (Cophenylcaine) nasal spray performs better than xylometazoline (Otrivine) spray for the purposes of out-patient rigid nasendoscopy preparation.

Design: Prospective, double-blind, randomised trial comparing visual analogue scores for out-patients receiving either phenylephrine-lignocaine mixture or xylometazoline, prior to undergoing rigid nasendoscopy as part of their assessment.

Subjects: Seventy-three patients requiring rigid nasendoscopy as part of their assessment were recruited to the study from Raigmore Hospital's out-patient clinic. These patients were randomised to receive a nasal spray comprising either phenylephrine-lignocaine mixture or xylometazoline, 10 minutes prior to rigid nasendoscopy. Double-blinding was adopted. After the procedure, the patient and the doctor independently completed separate visual analogue score-based questionnaires regarding the pain of the procedure and the ease of the examination, respectively.

Results: Analysis of the data using standardised statistical methods demonstrated that the phenylephrinelignocaine mixture did not perform better than xylometazoline, to any statistically significant extent.

Conclusion: Phenylephrine-lignocaine mixture is considerably more expensive and has potentially more side effects than xylometazoline. These study findings suggest that it is difficult to justify the use of phenylephrine-lignocaine mixture over xylometazoline, for nasal preparation prior to rigid nasendoscopy.
\end{abstract}

Key words: Nasal Cavity; Larynx; Endoscopy; Local Anaesthetic

\section{Introduction}

Rigid nasendoscopy is now considered essential for the comprehensive assessment of patients with sino-nasal symptoms. It can be an uncomfortable procedure for the patient, and it is important that mucosal oedema does not obscure the endoscopist's view. Topical nasal preparations which decongest the nasal mucosa to provide optimal viewing conditions are frequently used, often with the addition of a local anaesthetic. Cocaine was used extensively in the past, having both a decongestant and an anaesthetic effect, but it is a controlled drug and is therefore inconvenient to store and use in the clinic. Two modern alternatives are a phenylephrine-lignocaine mixture (Cophenylcaine; Aurum Pharmaceuticals, Romford, UK) and xylometazoline (Otrivine; Novartis Consumer Health, Horsham, UK). ${ }^{1,2}$ The former contains a decongestant and a local anaesthetic, whereas the latter contains only a decongestant. The added presence of lignocaine in the mixture is often assumed to reduce discomfort for the patient without compromising the examination. Both products are available as single-use preparations in UK ENT units. However, the phenylephrine-lignocaine mixture is significantly more expensive, at $£ 8.73^{3,4}(€ 12.88$ or US\$17.61), compared with xylometazoline, at $£ 1.42^{3}$ (€2.09 or US\$2.86). In addition, the phenylephrine-lignocaine mixture has some documented undesirable effects ${ }^{3}$ attributed to the presence of lignocaine, and indeed in some patients is contraindicated.

This study investigated whether phenylephrinelignocaine mixture is any better than xylometazoline when used as a nasal preparation prior to out-patient rigid nasendoscopy.

\section{Materials and methods}

A prospective, double-blind, randomised, controlled trial was designed. Sample size was based partly on a

From the Department of Otolaryngology/Head and Neck Surgery, Raigmore Hospital, Inverness, Scotland, UK.

Presented to the New Zealand Society of Otolaryngology, Head and Neck Surgery, $31^{\text {st }}$ October to $3^{\text {rd }}$ November 2006, Timaru, New Zealand.

Accepted for publication: 11 July 2008. First published online 13 October 2008. 
previous, similar study with similar methodology. ${ }^{4}$ A visual analogue scoring system of zero to 10 was adopted. It was felt reasonable that, for the purposes of this study, two or more points of difference on this scale would represent a significant difference. Less than two points of difference was considered not significant. Based on this, a sample size of 30 patients in each group was predicted to be necessary if the study was to have a chance of achieving its objective. This was on the basis of 80 per cent power with 95 per cent confidence. Thirty was also felt to be a reasonably achievable patient number in the context of a busy clinic in a district general hospital setting.

Seventy-three patients were recruited from the out-patient clinic at Raigmore Hospital between January 2004 and April 2005. All patients involved underwent a direct anterior nasal examination prior to recruitment.

The inclusion criteria comprised all adult patients (i.e. over 16 years of age) whose history and initial clinical findings indicated the need for rigid nasendoscopic examination. We excluded patients who were younger than 16 years, had previously undergone rigid nasendoscopy, were pregnant, had a previously documented allergic reaction to any of the medications, had poorly controlled hypertension, or had a nasal septal deviation obstructing the nasal airway by an estimated 50 per cent or more.

Patients were assigned to one of two groups, to receive either phenylephrine-lignocaine mixture or xylometazoline.

Randomisation was by means of pre-sealed, opaque envelopes with a label inside giving the group to which the patient would be assigned. Each envelope's label was produced by 'drawing from a hat' of 100 labels, 50 for each group. One hundred patient packs were made up prior to the commencement of the study. Each was numbered to represent consecutive patients recruited, so that there would be no risk of confusion regarding data collection and eventual analysis. Each patient pack contained an envelope with the group assignment label, a patient information sheet, patient and doctor questionnaires, and a consent form.

Once written, informed consent had been obtained, the patient was taken by a clinic nurse to a room separate from the endoscopist. Here, the nurse opened the envelope from that patient's pack to reveal their group assignment. The patient was not made aware of which spray was being used. The nurse then administered the relevant spray as a metered dose of two full pump sprays inside each nostril, encouraging the patient to nasally inhale as the medication was sprayed. Each patient was then asked to stay in the waiting area for 10 minutes. After this time, the endoscopist brought the patient to the examination room to perform rigid nasendoscopy. In this way, both the patient and the endoscopist were blinded to the spray administered.

Rigid nasendoscopy was standardised. A $30^{\circ}$, $2.7 \mathrm{~mm}$ endoscope with a portable light source was used. Both sides of the nose were examined, right side first. The endoscope was passed three times
TABLE I

PATIENT QUESTIONNAIRE

1 On a scale of 0 to 10 , give the score that you feel best describes the level of pain of having the telescope put inside the nose $(0=$ no pain, $10=$ worst ever pain $)$

2 On a scale of 0 to 10 , give the score that best describes the level of overall discomfort caused by what has been done today (includes the nasal spray as well as the telescope inside the nose $)(0=$ no discomfort, $10=$ most uncomfortable possible $)$

3 On a scale of 0 to 10 , after having the nasal spray, how anxious did you feel about having the telescope put inside your nose? $(0=$ not anxious at all, $10=$ most anxious possible)

4 On a scale of 0 to 10 , how happy would you be to have the entire procedure done again?

through the nose. Initially, this was along the floor of the nose to the nasopharynx, then at the level of the middle meatus, and lastly along the roof of the nose.

The endoscopist was either one of five consultants, an associate specialist, one of three specialist registrars or a senior house officer with at least one year's ENT experience.

After each rigid nasendoscopy was completed, both the patient and the endoscopist completed a separate visual analogue scoring questionnaire (Tables I and II). For this study, the measure of discomfort experienced by the patient provided the most useful information. Therefore, the patient's questions related mainly to scoring their discomfort, and the doctor's questions related to ease of procedure and efficacy of examination. Patients had the difference between scales explained to them, and a clinician or nurse was always available to answer any queries.

Data were found to be skewed to the lower part of the scale for questions relating to patient discomfort, and to the upper part of the scale for patient willingness to have the procedure repeated and ease of

TABLE II

DOCTOR QUESTIONNAIRE

1 On a scale of 0 to 10 , give the score that best describes the ease with which you were able to perform rigid nasendoscopy on the patient $(0=$ unable to perform procedure at all, $10=$ no problems at all $)$ Complete the following Table on the intra-nasal view obtained on rigid nasendoscopy, right side first (award a tick if structure was adequately visualised), and add up the total (maximum score $=8$ )

\begin{tabular}{lll}
\hline Features & Nostril & \\
\cline { 2 - 2 } & Right (1st) & Left (2nd)
\end{tabular}

Inferior turbinate

Nasopharynx

Middle turbinate

Middle meatus

Accessory ostia

Olfactory cleft

Ethmoidal bulla

Uncinate process

Total score 
examination. The data were processed using the Stata 8.0 statistical software package. Results were analysed using the Mann-Whitney U test for nonparametric data and the $t$-test for unequal variances.

The study had received the necessary review and approval from the local research ethics committee prior to recruitment. Participation in the study was on a voluntary basis for patients who would require rigid nasendoscopy as part of their assessment. The patients were provided with a patient information sheet, and nursing and medical staff were available at all times to answer any relevant questions.

\section{Results and analysis}

Complete data were collected from 61 patients - 34 women and 27 men. The mean scores for all outcome measures in each group are shown in Table III. No patient suffered side effects from either spray.

These results are summarised in Figures 1 and 2, demonstrating the main outcome criteria (patient questionnaire) and the secondary outcome measures (doctor questionnaire). Both showed that use of the phenylephrine-lignocaine mixture conferred an advantage, but this difference was not statistically significant.

\section{Discussion}

Our results appear to show that the phenylephrinelignocaine mixture was marginally better for nasal preparation prior to rigid nasendoscopy than xylometazoline, but not to a statistically significant extent. A study comparing these two commonly used medications, in this context, has not previously been published. Our findings are significant because the phenylephrine-lignocaine mixture contains two pharmacologically active agents while xylometazoline only has one. Although neither preparation carries a risk of significant complications, it is always desirable to use the most efficacious drug with the least potential side effects (in our study, no patients suffered significant side effects). The phenylephrine-lignocaine mixture is known to produce unpleasant local effects due to the presence of lignocaine, ${ }^{5}$ such as numbness, burning and bitterness. The phenylephrine-lignocaine mixture is considerably more expensive than xylometazoline when used as a once-only agent, as is the case in many otolaryngology units.

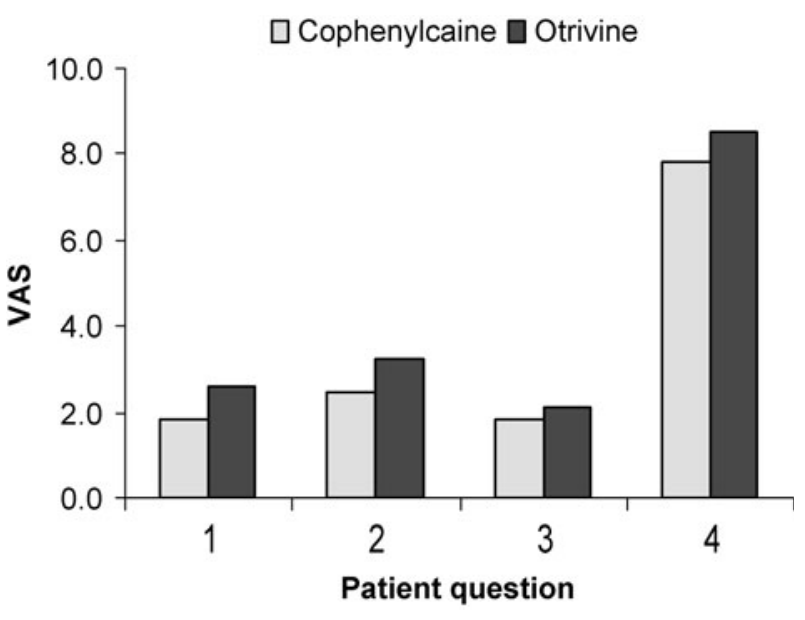

FIG. 1

Main outcome measures, i.e. patient questionnaire results.

Our study was prospective, double-blinded and randomised, and compared two commonly used preparations in a real life clinical setting.

To enable comparison of the two preparations, a relatively arbitrary point on the visual analogue scale was chosen as the boundary between clinical significance and non-significance. This was based on the opinions of those involved in the study and on the published literature reviewed. If less than two points of difference had been adopted as this boundary, then it is less likely that no significant difference would have been found with the patient numbers involved. Even if larger study numbers had resulted in a statistically significant difference, this would still not have been clinically significant in the context of our usual clinic numbers. However, it seems reasonable that those completing the questionnaires be expected to recognise a difference of two points on a ten-point visual analogue scale, but not necessarily less than two.

The study collected data on a total of 73 patients; however, for 12 patients the data were incomplete and therefore not included. It is possible that such data could have influenced the results in such a way as to alter the conclusions drawn, but this was considered to be unlikely.

Randomisation of grouping was determined by the main researcher, via random selection 'from a hat' prior to patient recruitment, rather than contemporaneously. Each predetermined grouping was

TABLE III

MEAN SCORES FOR ALL OUTCOME MEASURES IN EACH GROUP

\begin{tabular}{|c|c|c|c|c|c|c|c|}
\hline \multirow[t]{2}{*}{ Preparation } & \multirow[t]{2}{*}{ Pain } & \multirow[t]{2}{*}{$\begin{array}{l}\text { Overall } \\
\text { discomfort }\end{array}$} & \multirow[t]{2}{*}{ Anxiety } & \multirow[t]{2}{*}{$\begin{array}{c}\text { Would you have it done } \\
\text { again? }\end{array}$} & \multirow[t]{2}{*}{ Ease of examination } & \multicolumn{2}{|c|}{$\begin{array}{c}\text { Intra-nasal } \\
\text { structures } \\
\text { visualised }(n)\end{array}$} \\
\hline & & & & & & On R & On L \\
\hline $\begin{array}{l}\text { Phenylephrine-- } \\
\text { lignocaine }\end{array}$ & 1.96 & 2.47 & 1.62 & 8.41 & 7.10 & 5.06 & 4.97 \\
\hline Xylometazoline & 2.56 & 3.11 & 2.41 & 7.53 & 7.08 & 5.00 & 4.81 \\
\hline
\end{tabular}

$\mathrm{R}=$ right; $\mathrm{L}=$ left 


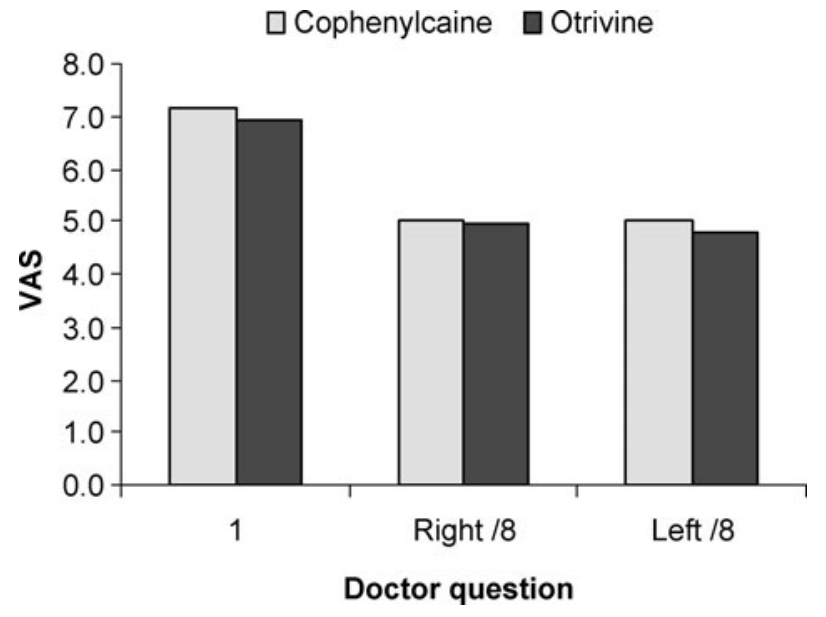

FIG. 2

Secondary outcome measures, i.e. doctor questionnaire results.

placed in a sealed envelope. These envelopes were then placed in individual, plastic-bound packs containing all the relevant paperwork for each patient recruited to the study. The packs were numbered consecutively to represent consecutive patients recruited. This was done: to improve the efficiency of study administration; to reduce any confusion at the time of recruitment in the context of a busy clinic; to reduce the risk of data being misplaced; and to make subsequent data collection easier. The researchers felt that the risk of incorrect conclusions due to inaccurately collected data, was greater than the bias risk due to pre-determined randomisation.

One could also argue that a third group of patients receiving no nasal spray should have been included in such a study. This might have allowed us to assess whether we needed to use anything at all. However, most otolaryngologists performing rigid nasendoscopy advocate the use of some form of topical nasal preparation. Therefore, such a group was not considered relevant or necessary, and in fact might have negatively influenced any valid conclusions drawn from the other two groups.

A literature review identified several studies investigating the use of topical nasal preparations for rigid nasendoscopy. ${ }^{1,2,6}$ Like our own study, all involved the use of a phenylephrine-lignocaine mixture and measured outcomes with visual analogue scales. Two of the studies compared its use with that of preparations other than xylometazoline. In one, ${ }^{1}$ it was found to compare more favourably to lignocaine in terms of operator ease and quality of view, with equivalent analgesia. Walshe et $a l^{6}{ }^{6}$ conducted a pilot study comparing a phenylephrine-lignocaine mixture with a cocaine solution and found no significant difference, again with the same outcome measures of pain and quality of view. This had important implications at that time because cocaine had been widely used for many years, was a controlled drug and had potentially serious side effects. The other study ${ }^{2}$ merely compared the timing of examination following administration of a phenylephrine-lignocaine mixture. It showed that its analgesic effect was better at 10 minutes than at 1 minute, a factor adopted in our own methodology.

- Phenylephrine-lignocaine mixture (Cophenylcaine) and xylometazoline (Otrivine) are popular nasal preparations used to improve the efficacy of rigid nasendoscopy and to reduce patient discomfort

- The phenylephrine-lignocaine mixture was perceived anecdotally to be better than xylometazoline, because it contained a local anaesthetic in addition to a decongestant

- This prospective, double-blind, randomised study demonstrated that, for such purposes, the phenylephrine-lignocaine mixture did not perform better than xylometazoline to a statistically significant extent

- On the basis of these results, there is limited justification for the use of phenylephrinelignocaine mixture rather than xylometazoline for out-patient rigid nasendoscopy preparation, given that phenylephrinelignocaine mixture is much costlier and has a greater risk of causing side effects

Two other studies involving a phenylephrine-lignocaine mixture compared it with cocaine for the purposes of flexible fibre-optic nasendoscopy. ${ }^{7,8}$ In these studies, it again compared favourably with cocaine. Cain et al. ${ }^{9}$ concluded that the use of phenylephrine-lignocaine mixture conferred no benefit over using no preparation at all. All these studies adopted similar methods and outcome measures as the studies involving rigid nasendoscopy. One study $^{5}$ compared xylometazoline to several preparations, including a phenylephrine-lignocaine mixture, for flexible nasendoscopy. The authors found, as did we, that xylometazoline was no less efficacious than phenylephrine-lignocaine mixture. Indeed, they concluded that any advantages conferred by the phenylephrine-lignocaine mixture were nullified by the unpleasant local effects (such as bitter taste) created by the lignocaine. In contrast to Cain et al., ${ }^{9}$ however, they concluded that the use of no preparation at all led to severe side effects.

\section{Acknowledgements}

The authors would like to thank S Craig and H Whyte, otolaryngology nurses, and S Selvaraj, regional statistician, without whom completion of this study would have been impossible. We would also like to thank the otolaryngology consultants at Raigmore Hospital for allowing their patients to be included in the study.

\section{References}

1 Douglas R, Hawke L, Wormald PJ. Topical anaesthesia before nasendoscopy: a randomised controlled trial of co-phenylcaine compared with lignocaine. Clin Otolaryngol 2006;31:33-5 
2 Pothier DD, Hall CEJ, Gillett S. Timing of co-phenylcaine administration before rigid nasendoscopy: a randomised, controlled trial. J Laryngol Otol 2006;15:1-3

3 Ears, nose and oropharynx. British National Formulary, RPS Publishing, 2008;12.2.3:589

4 Anaesthesia. British National Formulary, RPS Publishing, 2008;15.2:683

5 Sadek SAA, De R, Scott A. The efficacy of topical anaesthesia in flexible nasendoscopy: a double-blind randomised controlled trial. Clin Otolaryngol 2001;26:25-8

6 Walshe P, Rowley H, Hone S. Co-phenylcaine as an alternative to Brompton's solution in rigid nasendoscopy: a pilot study. J Clin Pharm Therap 2002;27:185-7

7 Latorre F, Otter W, Kleeman PP. Cocaine or phenylephrine/ lignocaine for nasal fibre-optic intubation? Eur J Anaes 1996; 13:577-81

8 Lennox P, Hern J, Birchall M. Local anaesthesia in flexible nasendoscopy. A comparison between cocaine and cophenylcaine. J Laryngol Otol 1996;110:540-2
9 Cain AJ, Murray DP, McClymont LG. The use of topical nasal anaesthesia before flexible nasendoscopy: a doubleblind, randomised controlled trial comparing cophenylcaine with placebo. Clin Otolaryngol 2002;27:485-8

Address for correspondence:

Mr N A McCluney,

54 Charles Street,

Aberdeen AB25 3TU, Scotland, UK.

Fax: 01224554569

E-mail: neilmccluney@doctors.org.uk

Mr N A McCluney takes responsibility for the integrity of the content of the paper.

Competing interests: None declared 